\title{
Perspective on thin film waveguides for on-chip mid-infrared spectroscopy of liquid biochemical analytes
}

\author{
Vinita Mittal $^{1 *}$, Goran Z. Mashanovich ${ }^{1,2}$ and James S. Wilkinson ${ }^{1}$ \\ ${ }^{1}$ Zepler Institute for Photonics and Nanoelectronics, University of Southampton, Southampton, SO17 1BJ, United Kingdom \\ ${ }^{2}$ School of Electrical Engineering, University of Belgrade, 11120 Belgrade, Serbia \\ *Email: V.Mittal@soton.ac.uk
}

\begin{abstract}
Miniaturized spectrometers offering low cost, low reagent consumption, high throughput, sensitivity and automation are the future of sensing and have significant applications in environmental monitoring and food safety, biotechnology, pharmaceuticals and healthcare. Mid-infrared (MIR) spectroscopy employing complementary metal oxide semiconductor (CMOS) compatible thin film waveguides and microfluidics shows great promise towards highly integrated and robust detection tools and liquid handling. This perspective provides an overview of emergence of thin film optical waveguides used for evanescent field sensing of liquid chemical and biological samples for MIR absorption spectroscopy. The state of the art of new material and waveguide systems used for spectroscopic measurements in the MIR is presented. An outlook on the advantages and future of waveguide-based MIR spectroscopy for application in clinical settings for point-of-care biochemical analysis is discussed.
\end{abstract}

Bodily fluids such as blood serum/plasma, urine, saliva, cerebrospinal fluid and tears hold crucial information on a variety of proteins and biochemical products, thereby providing important information on a person's health. Moreover, these bodily fluids are important alternative samples to cells and tissues for disease diagnosis and prognosis, allowing liquid biopsy, for example, with advantages of minimal invasiveness, rapid sample collection with minimum cost/discomfort and sample processing [1]. The study of bodily fluids to reveal specific biochemical information contained in samples is therefore of increasing importance. Given the large population suffering from various kinds of physical and mental diseases, healthcare systems are under a considerable load for diagnostics and treatment. Ideally, patients should be screened by a non-expert using affordable diagnostic tests before referring patients to experts. Affordable tests are required for screening of masspopulation not only for diagnostics but also for drug design and development. There are several techniques extensively employed to study bodily fluids such as microscopy, chromatography, fluorescence staining, mass-spectrometry, nuclear magnetic resonance, and vibrational spectroscopy based on Raman and mid-infrared (MIR) absorption. Each technique has its own advantages and shortcomings and they can be applied in combination with each other for improved confidence.

This perspective focusses on the study of aqueous samples on thin film waveguides using the promising non-destructive vibrational spectroscopic technique of MIR absorption spectroscopy. Optical waveguide approaches have several advantages compared to bulk liquid absorption spectroscopy including miniaturized design, high sensitivity for small sample volume, precise optimization for signal to background interferences, ready on-chip integration with microfluidics and simple operation. The body mass of adult humans is $50-60 \%$ water so that chemical processes in the human body take place in aqueous solutions. This presents a challenge for MIR spectroscopy as water absorbs light strongly at some MIR wavelengths. The aim of this study is to review progress on thin film waveguides used for MIR absorption spectroscopy of biochemical and other aqueous samples. Thin film waveguide technologies for MIR spectroscopy are still in their early stages of research and there are a limited number of examples of biochemical analyte demonstrations to date. Therefore, this perspective includes studies of several non-biochemical liquid analytes to allow good comparisons of competing waveguide technologies. It also summarizes the state of the art on MIR materials and thin film waveguide sensors to explore the potential of MIR waveguide spectroscopy for measurement of body fluids for clinical applications.

Optical sensors based on thin film waveguides offer compact, portable and scalable multi analyte detection sensing with low analyte volume needed for the measurement. A large number of sensors can be integrated on a wafer using microfabrication mass manufacturing, allowing affordable and multiplexed sensors and potentially enabling integration with electronics read out circuitry and microfluidics on the same chip. While most MIR demonstrations of waveguide absorption spectroscopy to date have analyzed bulk liquid media, waveguide sensors are of particular benefit when measuring ultrathin layers where strong interaction between a biological monolayer/bilayer and light can be maintained over centimeters of length of the waveguide. Centimeter-long path lengths can be achieved on small chips by introducing engineered bends and spirals [2, 3]. The sensitivity of the waveguides can be further increased by using suspended waveguides where a large fraction of evanescent field lies outside the structure and the waveguide core can be surrounded by analyte. 

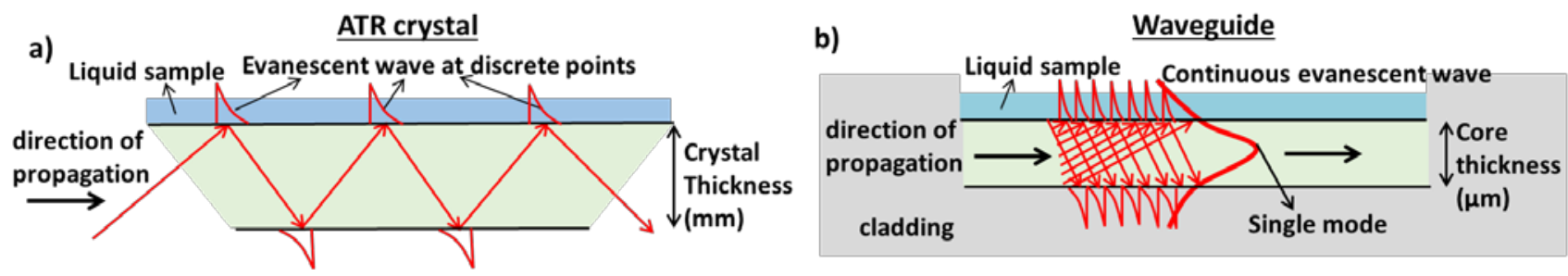

Figure 1 Schematic of light propagation in a) an ATR crystal and b) an optical waveguide

IR spectroscopy is a non-destructive, label-free, highly specific technique used for identification of molecular compositions and their structures by providing their unique fingerprints. It yields direct information on molecular vibrational transitions and provides inherent molecular selectivity without the need for additional labels such as chemical dyes, saving time, cost and complexity. Laboratory studies of the MIR absorption spectra of liquid samples typically use a Fourier transform infrared (FTIR) spectrometer with the sample in a cuvette made of IR transparent material and illuminated by white light from a "glo-bar". As the MIR region is dominated by strong background water absorption, for aqueous samples the cuvette pathlength is limited to a few tens to hundreds of microns [4], rendering cuvettes difficult to use as they tend to clog. The use of attenuated total reflection (ATR) crystals combined with FTIR avoids this problem and, being an evanescent field technique, suffers less from interferences from the bulk of the liquid such as scattering from biological cells.

An ATR element is in effect a few millimeter thick highly multimoded waveguide in which the input ray is trapped by total internal reflection and "bounces" off the top surface at a few discrete locations, at which the resultant evanescent field interacts with the liquid sample, as shown in Figure 1 (a). Each reflection yields absorption, through penetration of the evanescent field into the sample by only the equivalent of a few microns cuvette thickness, so that many reflections and large surface coverage are needed to achieve a strong absorption and hence a clear and quantifiable spectral signature.

Recently, a micro-groove silicon (Si) ATR has been demonstrated as cost-effective, flexible and single time use alternative to bulk Si ATR [5]. Two exemplary analytes of potassium carbonate and sodium acetate were used to evaluate and compare the analytical performance of the micro-groove Si ATR with commercially available Si ATR using FTIR and Quantum Cascade Lasers (QCL) in the MIR spectral region of $5.5 \mu \mathrm{m}$ to $16.6 \mu \mathrm{m}\left(1800 \mathrm{~cm}^{-1}\right.$ to $\left.600 \mathrm{~cm}^{-1}\right)$. In another example, a diamond ATR has been used with the QCL covering a spectral region of $4.95 \mu \mathrm{m}$ to $11.2 \mu \mathrm{m}\left(2020 \mathrm{~cm}^{-1}\right.$ to $\left.890 \mathrm{~cm}^{-1}\right)$ for monitoring glucose in human saliva [6].

Thin film waveguides are essentially ultrathin ATR elements in which the discrete reflections become a continuous evanescent wave along the waveguide surface as shown in Figure 1 (b). These thin film waveguides comprise a high refractive index guiding layer supported on a low refractive index substrate with an evanescent wave extending into the upper cladding. The resultant continuous evanescent wave provides much higher absorption efficiency (per unit length of sample) than a conventional ATR element. Waveguides also offer compact designs with an added benefit of potential integration of reference arm to avoid contributions from drift and noise by allowing simultaneous measurement of reference and the sample analyte. While such waveguides do not in themselves re- move the problem of competing water absorption, they do offer precise tailoring of the exact interaction length to optimize absorption and hence signal-to-noise ratio (SNR).

\section{BACKGROUND WATER ABSORPTION IN BIOLOGICAL FLUIDS}

There has been speculation whether MIR spectroscopy can measure low concentration species in aqueous analytes effectively, due to the dominance of water absorption which limits the useable pathlength and hence the potential sensitivity. Water absorption can be reduced by drying the samples before measurement, but this procedure adds time and cost and yields unrepresentative absorption spectra. This is a particular issue for protein structure measurement as proteins change their conformation and structure on drying, resulting in their physical and chemical degradation, and leading to inaccuracies in the interpretation of results $[7,8]$. Methods to minimize the effect of water absorption are being pursued. One such method is liquid-liquid extraction [9, 10], where two liquids are brought together in one microfluidic channel using laminar flow lines, molecules diffuse from the aqueous to the nonaqueous medium and, finally, separation of liquids takes place based upon their miscibility and surface tension. An optofluidic device has been designed with hydrophobic and hydrophilic surfaces to modify the wetting properties of liquids as shown in Figure 2 a) [9]. Cocaine was extracted from saliva in perchloroethylene (PCE) using liquid-liquid extraction in the microfluidic system bonded onto the germanium on silicon waveguide shown in Figure 2 b) and optically characterized. This sophisticated way to separate liquids may be applied where the analyte volume is available in milliliter-order quantities, but it requires the additional separation step and hence time.

Another way to minimize dominance of the water absorption is by modifying the waveguide surface using a recognition layer to enrich the target analyte molecules for higher selectivity, sensitivity and also to reduce water absorption in the analytical volume probed by the evanescent field [11, 12]. For example, in reference [11], proteins and polypeptides were immobilized by chemically attaching chelating nitrilotriacetic acid (NTA) groups on the surface of Ge and ZnSe ATR elements. After that, the modified surfaces were activated by transition metal ions to enable them to selectively bind polyhistine sequences within the proteins of interest. Two strategies were employed to cover the ATR surfaces with NTA groups: 1) Self-assembly of a chelatorthioalkane (CTA) on Au-coated surfaces and 2) Silylation of $\mathrm{SiO}_{2}$-coated surfaces with a mercaptosilane followed by reaction with NTAmaleimide. The authors concluded that the binding process was reversible and on comparison, the silanized surface produced better reproducibility and twice the signal to noise ratio (SNR) whereas CTA-coated gold surfaces enhanced the 

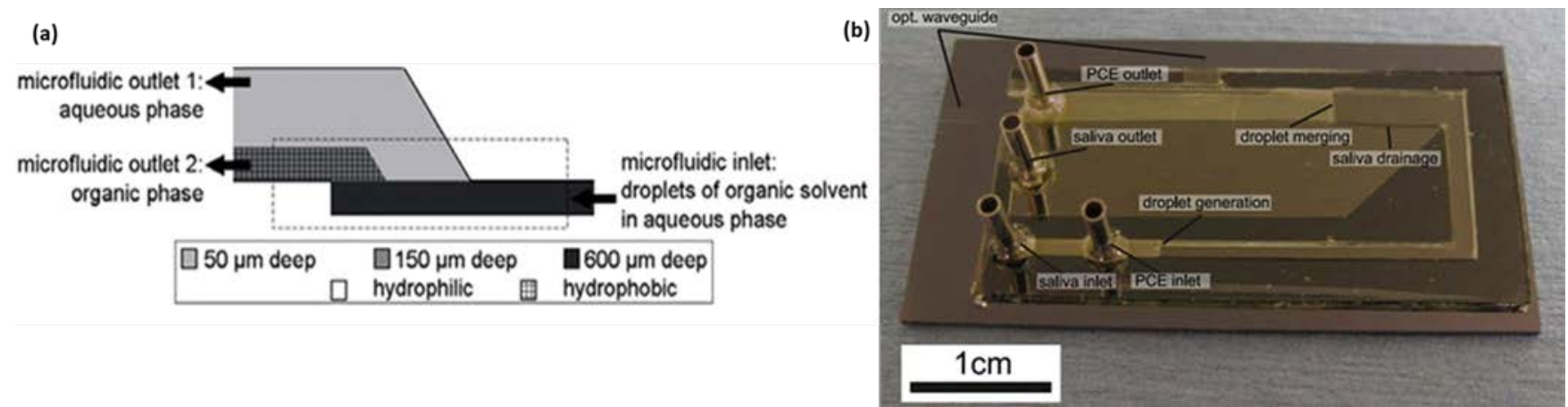

Figure 2 a) Scheme of the microfluidic merging geometry with different channel depths and surface properties and b) Polymer microfluidic part bonded onto the optical waveguide device. Reprinted with permission from [7]. Copyright (2013) American Chemical Society.

surface sensitivity by an order of magnitude. In reference [12], surface recognition layers on waveguides using sol-gel process is proposed to reduce the effect of water absorption. The pore size of the sol-gel based coatings can be controlled to maximize their surface area in order to ensure high diffusion rates into and out of the sol-gel films, thereby tuning their surface hydrophobicity. So far, this technique has been implemented for chemical recognition layers, however, the authors suggest it can be applied for biomedical applications.

In the next section, state of the art on various thin film waveguide systems used for aqueous biochemical samples is discussed.

\section{MIR EXPERIMENTAL APPARATUS}

Broadly, the experimental apparatus used for on-chip sensing of aqueous analytes comprises a laser system emitting MIR light (pulsed or continuous), MIR components such as lenses and fibers to focus the light in and out of the waveguide and an MIR detector to measure the signal. A schematic of a typical MIR experimental apparatus is shown in Figure 3. The magnified portion shows the interaction of an analyte with the evanescent field of the waveguide mode. MIR sources, detectors, fibers and lenses are briefly discussed in the following sections.

\section{MIR Sources}

Historically, the broadband thermal emitters used in FTIR were the predominant light sources employed at MIR wavelengths. However, these sources were inefficient and incompatible with narrow-band, highly directional and high-power applications. In the mid-1990s, the landscape of MIR light sources changed with the advent of Quantum Cascade Lasers (QCLs), that operate on the principle of inter sub-band transitions between the engineered conduction bands of semiconductor heterostructures. QCLs enabled compact, roomtemperature, high power, pulsed and continuous wave operation with the flexibility to tune the MIR wavelength. A QCL is in many ways an ideal light source for many MIR applications, however, the $\sim$ ps timescale for non-radiative recombination lifetime associated with its inter sub-band optical transitions lead to large threshold current densities that limit its wall-plug efficiency [13,14]. To overcome this shortcoming, the Interband Cascade Laser (ICL) was introduced in 2008. The ICL emits MIR light by recombination of electrons in conduction band quantum well (QW) and holes in valence band QW in complex heterostructures. ICLs are particularly promising in the 3-7 $\mu \mathrm{m}$ wavelength region. The low current density of ICLs offers MIR applications the potential for low power, handheld or battery-operated systems. However, it is challenging to operate ICLs at high powers as temperature fluctuations alter their performance. QCLs offer higher slope efficiencies, higher peak powers and less temperature dependence in comparison with ICLs. QCLs and ICLs are the two competing technologies for tunable MIR laser sources today. QCLs offer high brightness and therefore higher power coupling efficiency into waveguides, allowing longer pathlengths with improved signal to noise ratio and hence a stronger absorption signal. Therefore, the use of high power QCLs with waveguides may be the most economical and powerful combination in the future. The next generation MIR systems require simple cost-effective diode-like sources similar to the visible and near-infrared devices, with good efficiency, room temperature operation and compact integrable systems. Their appeal is in their simple modelling, design and growth, energy efficiency and operation. Some other potential future MIR source technologies include 1D heterostructures, Quantum Wells, Quantum dots (QD), type II superlattices using various material systems such as GaAs, InP, InAs, lead salts and many more. These future technologies depend on the quantum engineering of electronic states of complex heterostructures with the investigation of sub-diffraction limited optical confinement of carriers and reaching the extremes of light matter interactions in the MIR region [13, 15 - 17].

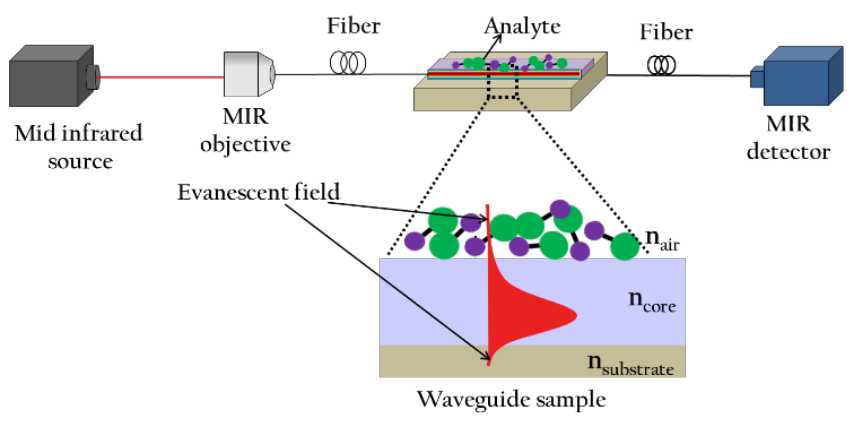

Figure 3 Typical waveguide absorption spectroscopy system

\section{MIR Detectors}

There are two main types of IR detector: thermal and photonic. Thermal detectors comprise thermopiles, thermocouples and bolometers where the sensing mechanism depends on thermal phenomena due to the incident IR beam. Thermocouples and thermopiles operate on thermoelectric phenomena 
whereas bolometers are based on change in resistance of the sensor material due to IR beam falling on it. Photonic detectors comprise photoconductive and photovoltaic detectors. Here the sensing mechanism is based on electronic excitations due to incident IR beam $[18,19]$. There are few main parameters that are important when comparing the detectors: volume, weight, cost and performance. Thermal detectors operate at room temperature and are low cost, low performance and high volume, whereas MIR photonic detectors have high cost, high performance, and low volume and normally need cryogenic cooling for their operation. The early detection technology was developed in 1940s based on compound semiconductors namely, PbS, PbSe, PbTe, InSb and mercury cadmium telluride $(\mathrm{MCT})$.

The current technology is mainly focused on photoconductive MCT because it covers a wide IR wavelength range (as high as $\sim 20 \mu \mathrm{m}$ ), is highly sensitive and has a fast response [15]. Its detection mechanism is based on the interband transitions between conduction and valence band of the ternary alloy of CdTe and HgTe. Despite being a high cost technology, MCT detectors dominate the MIR detector market due to their good performance. Another promising detector technology is the quantum well infrared photodetector (QWIP), where the detector material is AlGaAs/GaAs and the detection mechanism is based on inter sub-band transitions[20, 21]. QWIPs cover a wide range of IR wavelengths and are low cost as compared to MCT, however, they suffers from low quantum efficiencies and require lower operating temperatures than MCT. Some upcoming IR detector technologies include the quantum dots infrared photodetector (QDIPs) [22, 23] and type II strained super lattices [22, 24] similar to IR sources.

\section{IR fibers and lenses}

Commonly used IR fibers are based on fluorides and chalcogenides. Both single mode and multimode fibers made of $\mathrm{CaF}_{2}, \mathrm{InF}_{3}, \mathrm{ZrF}_{4}$, AsS, AsSe and alloys of sulfides and selenides are commercially available [25,26]. Fluoride fibers are used for applications requiring short wavelength range (up to $\sim \lambda=5.5 \mu \mathrm{m}$ ), whereas chalcogenide fibers can transmit longer into the MIR region (up to $\sim \lambda=16 \mu \mathrm{m}$ ). There are also hollow core fibers with the transmission window of $\lambda=2-16 \mu$ m [27]. The main concern with fibers is their handling as they are extremely brittle, and also the fiber losses. To overcome these limitations, IR transmitting lenses are frequently used for both input and output coupling. Some of the commercially available lenses are made of $\mathrm{CaF}_{2}$, Si, Ge, $\mathrm{ZnSe}$ with appropriate antireflective coatings [25, 28].

\section{THE STATE OF THE ART OF THIN-FILM MIR WAVEGUIDES}

The state of the art shows that many different waveguide materials can be used for waveguide spectroscopy in the MIR. There are several key parameters to be considered when choosing an appropriate waveguide material, depending on the application, such as: 1 . Transparency in the wavelength region of interest, 2. High refractive index contrast between waveguide core and surrounding media to allow high surface intensity and confinement of light into small geometries. 3. Chemical compatibility of the waveguide material with the analyte, in order not to change either the waveguide surface or the analyte composition, structure or orientation, 4. Ease of fabrica- tion, 5. Low optical propagation losses and low coupling and reflection losses (related to both materials and waveguide design) for high $\mathrm{S} / \mathrm{N}$ ratios, and 6. Mechanical strength.

MIR evanescent spectroscopy has been demonstrated on several waveguide systems, usually with studies on common solvents to establish proof of principle and to validate operation before proceeding towards biological fluids for clinically relevant applications. Various MIR transparent material systems have been explored to form appropriate MIR waveguides. The following section is divided into six subsections according to the waveguide material system used for sensing. It briefly describes the fabrication and characterization methods employed, the type of analyte used and, where available, the limit of detection (LOD) obtained in specific wavelength regions.

\section{Silver halides}

The first MIR evanescent field absorption-based sensing of liquid-phase analytes using free standing planar silver halide ( $\mathrm{AgC}_{10.4} \mathrm{Br}_{0.6}$ ) waveguides was demonstrated in 2005 [29], with a distributed feedback QCL operating in pulsed mode. Here thick films (190-300 $\mu \mathrm{m}$ thick and 3mm wide) of silver halide were developed from cylindrical fiber segments using presstapering method to form multi-mode waveguides. QCL emission at two frequencies was used: $1650 \mathrm{~cm}^{-1}(\lambda=6.06 \mu \mathrm{m})$ to detect amide peaks in solid urea crystals precipitated at the waveguide surface and the frequency of $974 \mathrm{~cm}^{-1}(\lambda=$ $10.26 \mu \mathrm{m})$ to detect $\mathrm{CH}_{3}-\mathrm{C}$ absorption feature of acetic anhydride that was dissolved in acetonitrile. Transmission measurements were carried out using an MCT detector for both the analytes deposited on the waveguides and compared with the spectra collected from a commercial FTIR using external assembly where light from the FTIR was focused onto the endfacet of the waveguide using a gold-coated off-axis parabolic mirror and the output signal was recorded onto the MCT detector. It was found that for $10 \mu \mathrm{L}$ droplets of urea deposited on the waveguide which is equivalent to $0.32 \mathrm{mg}$, an LOD of $80.7 \mu \mathrm{g}$ was achieved using the QCL-waveguide set-up, which was a factor of 4 higher than that achieved during the corresponding FTIR-waveguide measurements. For $1 \mu \mathrm{L}$ acetic anhydride droplets deposited on the waveguides, the LOD achieved using the QCL-waveguide system was $0.01 \mu \mathrm{L}$ $(10.8 \mu \mathrm{g})$ which was similar to that achieved using the corresponding FTIR-waveguide set-up. The reason for obtaining a similar LOD for acetic anhydride was that the $974 \mathrm{~cm}^{-1}$ frequency overlaps with the side mode of acetic anhydride and the authors suggests a significant improvement in its detection could be achieved if a laser emitting at $990 \mathrm{~cm}^{-1}(\lambda=10.1 \mu \mathrm{m})$ was used, corresponding to its fundamental absorption frequency. In 2013, the same group designed and fabricated grating couplers using focused ion beam milling on a planar silver halide waveguide of dimensions $20 \times 3 \times 0.3 \mathrm{~mm}$ [30]. Efficient coupling of light into the waveguides from QCL emitting at $10.4 \mu \mathrm{m}$ wavelength was confirmed by analyzing acetic acid mixed in water at different concentrations $(0,25,50,75$ and $100 \%$ ) by dropping $0.5 \mu \mathrm{L}$ of liquid on the waveguides. QCL output signal dampening was reported by recording the power of single laser pulse with different concentrations of acetic acid and a calibration curve was generated. Although halides have wide transparency in the MIR (3-20 $\mu \mathrm{m})$, they are not suitable for aqueous samples as they are hygroscopic and have limited stability, and they are difficult to grow as thin films. 

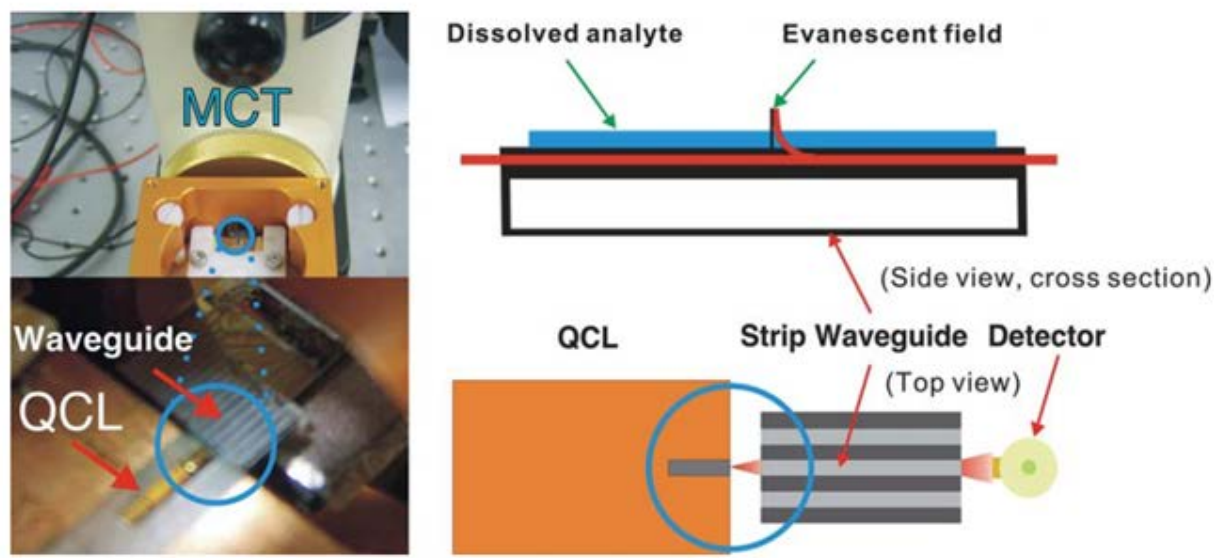

Figure 4 Experimental IR sensor apparatus combining a QCL with a GaAs/AlGaAs strip waveguide chip and a MCT-liquid N2 cooled detector. Reprinted with permission from [32] Copyright (2012) Royal Society of Chemistry.

\section{Gallium Arsenide (GaAs)}

In 2006, detection of acetic anhydride using a QCL emitting at $\lambda=10.3 \mu \mathrm{m}$ and a GaAs waveguide was demonstrated [31]. GaAs was deposited by molecular beam epitaxy (MBE) on a $\mathrm{Si}$ - doped GaAs substrate with a bottom cladding of AlGaAs in between. The GaAs strip waveguides were $500 \mu \mathrm{m}$ wide and $6 \mu \mathrm{m}$ thick. Here a $0.5 \mu \mathrm{L}$ drop of acetic anhydride was deposited on the waveguide surface covering a length of $3 \mathrm{~mm}$ at the center of the waveguide. Similarly, two or more droplets of the same volume and surface area were added sequentially on the waveguide surface to generate a response curve corresponding to the change in light intensity as a function of absorbing analyte path length. The same experiment was repeated with the multimode silver halide waveguide used previously in [29] and the results were compared. An improvement by a factor of 5 in sensitivity was achieved using single mode GaAs waveguides over multi-mode silver halide waveguides. Later, in 2012, the same group demonstrated an LOD of 19.4ng (18pL) for acetic anhydride when they used a narrower (200 $\mu$ m wide) GaAs waveguide made by metal organic vapor phase epitaxy (MOVPE) [32]. In the same publication they demonstrated a further reduction in the waveguide width to $50 \mu \mathrm{m}$ resulting in an improved LOD of $0.05 \mathrm{pL}$. The experimental set-up used for the measurement is reproduced in Figure 4. With the same waveguide material system, this group reported the first functional demonstration of MIR Mach-Zehnder interferometers (MZI) covering the wavelength region of 5.78-6.35 $\mu \mathrm{m}$ [33]. They tested the device by depositing droplets of water at different places of MZI and recorded the signal. In 2016, the performance of GaAs thin film waveguide technology combined with QCLs for MIR spectroscopy of various analytes was compared with the conventional single bounce diamond ATR crystal combined with FTIR technology [34]. GaAs waveguides chips of dimensions $5 \mathrm{~mm} \times 10 \mathrm{~mm}$ were cleaved and placed into a polytetrafluoroethylene (PTFE) inlay, which was in turn mounted into a stainless steel plate enclosure. Ammonium perchlorate dissolved in water was studied at $1100 \mathrm{~cm}^{-1}$ for the stretching vibration of $\mathrm{ClO}_{4}-$. The LOD for ammonium perchlorate was found to be $62.4 \mathrm{mM}$ for the $10 \mu \mathrm{m}$ wide GaAs waveguide, $56.9 \mathrm{mM}$ for the $6 \mu \mathrm{m}$ wide $\mathrm{GaAs}$ waveguide and ATR-FTIR spectrometer yielded $63.3 \mathrm{mM}$. Similarly, benzaldehyde solution was investigated in diethylene glycol monoethyl ether (DGME) at $1700 \mathrm{~cm}^{-1}$ to study the stretching vibration of $\mathrm{C}=\mathrm{O}$ and LOD for $6 \mu \mathrm{m}$ wide wave- guide was found to be $35.1 \mathrm{mM}$ and for ATR-FTIR LOD was determined to be $46 \mathrm{mM}$. Broadband detection of ammonium perchlorate solutions were also analyzed in the wavelength region between $1150-1050 \mathrm{~cm}^{-1}$ and it was observed that difference between 0.01 and $0.05 \mathrm{M}$ solutions could still be discriminated adequately. In the same year (2016), GaAs waveguides were integrated with a miniaturized liquid flow cell for continuous trace analysis of chlorinated hydrocarbons (CHCs) in water to ensure water quality [35]. A 1wt\% ethylene propylene copolymer (EPCo) membrane was deposited on the waveguide surface and optimized for reproducibility of hydrocarbon enrichment. As a model analyte for CHCs, a diluted perchloroethylene (PCE) in DI water was measured at various concentrations using a QCL and at $910 \mathrm{~cm}^{-1}$ an LOD of $5 \mathrm{ppm}$ was obtained. Further a mixture of PCE and trichloroethylene (TCE) was measured in the spectral range between 950 and $890 \mathrm{~cm}^{-1}$. The characteristic absorption peak of PCE was observed at $910 \mathrm{~cm}^{-1}$ and that of TCE was observed at $930 \mathrm{~cm}^{-1}$. In 2017, mycotoxin contamination in food was determined using GaAs waveguides combined with a QCL emitting at 1560$1820 \mathrm{~cm}^{-1}[36]$.

GaAs has refractive index close to $\sim 3$ and wide transparency in the MIR region up to $13 \mu \mathrm{m}$, however, it requires costly epitaxial techniques to deposit films on substrates with similar lattice constant to minimize stress. The substrate is generally an alloy of GaAs with a refractive index close to that of the waveguide core, so that thick films are needed, resulting in expensive and time-consuming fabrication, and weaker evanescent interaction. Moreover, GaAs is not biocompatible and can release toxic substances such as $\mathrm{As}$ or $\mathrm{AsO}_{\mathrm{x}}$ into aqueous analytes which can, for example, lead to cell death [37, 38].

\section{Chalcogenides}

Planar waveguides of mercury cadmium telluride (MCT) were fabricated as on-chip MIR transducers and evanescent field sensing of acetone was demonstrated by depositing a drop of solution on the waveguide [39]. The MCT waveguides were coupled with a QCL emitting in the spectral range 5.78$6.35 \mu \mathrm{m}$ via a ZnSe lens and the output light from the waveguide was coupled to an MCT detector using a pair of ZnSe lenses. The LOD of acetone mixed in isopropanol was calculated to be $1 \mathrm{~nL}$ (791ng). Chalcogenide glass made of GeAsSe was thermally evaporated to obtain thin films and the wave- 
guides were fabricated using photolithography and dry etching using fluoride chemistry [40]. Fluoropolymer films of different thicknesses were deposited on the waveguide core to protect the waveguide from surface contaminants, and the waveguides were optimized for lowest losses with respect to fluoropolymer thickness. The experimental set-up consisted of an optical parametric amplifier (OPA) and QCL's covering the wavelength range of $3-7.5 \mu \mathrm{m}$ and the coupling of light in and out of the waveguide was achieved using chalcogenide lenses and an IR camera, and a detector was used to record the signal. A fluoropolymer coating of thickness $10 \mathrm{~nm}$ yielded the lowest loss of $0.3 \mathrm{~dB} / \mathrm{cm}$ for $5 \mu \mathrm{m}$ wavelength. Sensing of a $0.1 w t \%$ solution of Prussian blue (PB) dissolved in dimethyl sulphoxide (DMSO) was demonstrated on the waveguide system. The resultant absorption spectra showed the absorption peaks of $\mathrm{PB}$ including the main $\mathrm{C}-\mathrm{N}$ stretching vibration band at $\lambda=4.775 \mu \mathrm{m}$.

A Ge-Sb-Se thin film based sensor was demonstrated to detect organic molecules in water at $\lambda=7.7 \mu \mathrm{m}[41,42]$. The ridge waveguides were $8-14 \mu \mathrm{m}$ wide and $1.7 \mu \mathrm{m}$ thick. Surface functionalization of the waveguides was achieved by using polyisobuthylene (PIB) polymer to extract small aromatic hydrocarbons such as benzene, toluene and xylene. The use of hydrophobic polymer is an effective method to increase the concentration of analytes at the waveguide surface, however, it is limited by polymer thickness as the sensitivity and detection limit of the sensor will decrease with increase in polymer film thickness [43]. The theoretical LOD for toluene dissolved in water was estimated to be $26 \mathrm{ppb}$ which was lower than the maximum level suggested by international standards of quality of water.

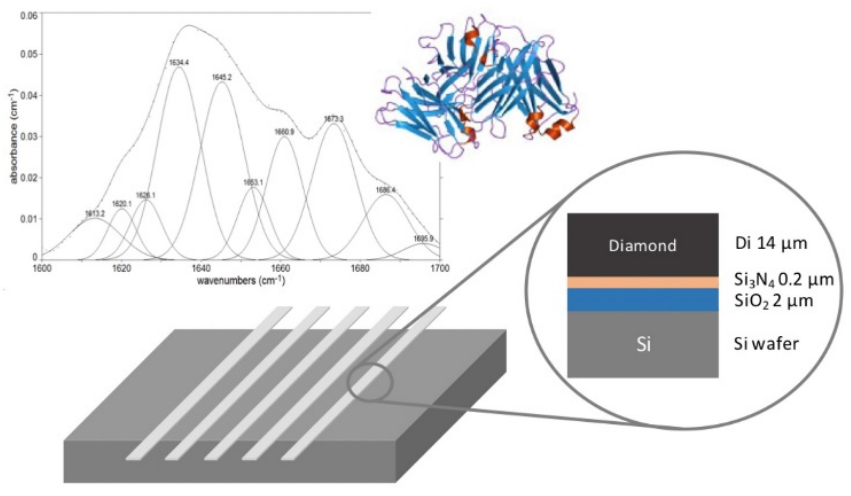

Figure 5 Diamond strip waveguides grown on Si showing the myoglobin protein amide I band with curve fitting. Reprinted with permission from [50] Wiley-VC (2016).

On-chip MIR absorption spectroscopy of ethanol in cyclohexane at $\lambda=5.2 \mu \mathrm{m}$ was demonstrated using chalcogenide glass $\left(\mathrm{Ge}_{23} \mathrm{Sb}_{7} \mathrm{~S}_{70}\right)$ micro disk resonators [44]. A drop in extinction ratio was observed with increase in concentration of ethanol and a wavelength shift in resonance peaks were also observed attributed to change in refractive index of the mixture at different concentrations. In 2018, ZnSe rib waveguides were fabricated on oxidized silica to demonstrate spectroscopy of IPA in water in the wavelength range of 2.6-3.7 $\mu \mathrm{m}$ [45]. $3.4 \mu \mathrm{m}$ thick $\mathrm{ZnSe}$ waveguides were deposited by thermal

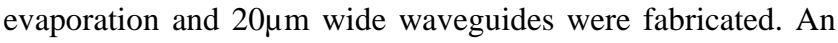
OPO was used as a light source and fluoride fibers were used for input and output coupling from the waveguide. A TE cooled MCT detector was used to record the transmission spectra. Here simple paper fluidics using filter paper was utilized to conduct the liquid to the waveguide and to define the evanescent path-length of the liquid. Mixtures of IPA in water $(0,20,40,60,80$, and $100 \%)$ were measured and compared with a theoretical waveguide based numerical model.

Chalcogenide materials have a wide transparency window from visible wavelengths to $20 \mu \mathrm{m}$ and have high refractive indices, according to their composition. They can be deposited into thin films with commonly used deposition techniques. However, these materials are soft and brittle, so their handling can be difficult. These materials also react with strong acids and bases, for example ZnSe is limited to use with samples with $\mathrm{pH}$ between 5 and 9 [46]. While the $\mathrm{pH}$ of blood, saliva and spinal fluid is about 7.4, the $\mathrm{pH}$ in stomach ranges between 1.5-3.5 and that of urine is between 4.5-8.0 [47, 48].

\section{Diamond}

The combination of thin film diamond waveguides with a QCL has been used to demonstrate spectroscopy of acetone in $\mathrm{D}_{2} \mathrm{O}$ [49]. Diamond waveguides were grown using chemical vapour deposition (CVD) on a passivated silicon substrate with a silicon nitride layer added to promote adhesion. $100 \mu \mathrm{m}$ wide and $14 \mu \mathrm{m}$ thick diamond waveguides were fabricated using photolithography and dry etching. An LOD of acetone in $\mathrm{D}_{2} \mathrm{O}$ of 200pL (158ng) was achieved. Broadband detection of anisaldehyde was also measured on $150 \mu \mathrm{m}$ wide diamond waveguides showing its two characteristic absorption peaks at 1596 and $1681 \mathrm{~cm}^{-1}$. Later, $500 \mu \mathrm{m}$ wide diamond waveguides were used to study the secondary structure of proteins using a QCL and the results were compared with those obtained from a 10-bounce diamond ATR combined with the FTIR system [50]. The amide I band of three proteins: bovine serum albumin, $\gamma$-globulin and myoglobin dissolved in phosphate buffer saline (PBS) were studied in the wavelength region of 1700$1600 \mathrm{~cm}^{-1}$ (Figure 5). The peak positions of secondary structures found in all three proteins were in agreement with literature studies carried out on a silicon ATR. A $5 \mu \mathrm{L}$ sample of concentration $10 \mathrm{mg} / \mathrm{mL}$ was used for the measurements on diamond waveguide and $20 \mu \mathrm{L}$ of a $0.5 \mathrm{mg} / \mathrm{mL}$ of sample was used on the ATR crystal for comparison. The amide I absorption peak was deconvolved to fit the secondary structures such as $\alpha$-helix and $\beta$-sheets on BSA and $\gamma$-globulin. Differences in the peak shape were observed between the diamond waveguide measurements and ATR measurements, for both proteins, and these were attributed to differences in the characteristics of the light sources and the waveguides. A 4-fold sensitivity gain was observed with QCL-diamond waveguides compared to the diamond ATR/FTIR apparatus. The same diamond waveguides used in [40] were also tested for N-Ndimethylformamide (DMF) in water, where an LOD of 5 vol\% was derived at $\lambda=6.06 \mu \mathrm{m}\left(1650 \mathrm{~cm}^{-1}\right)$ [51]. 
(a)

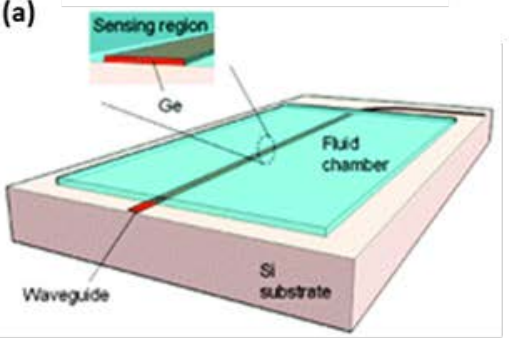

(b)

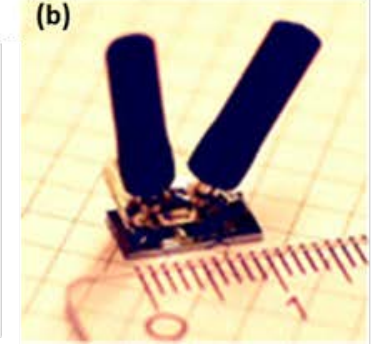

(c)

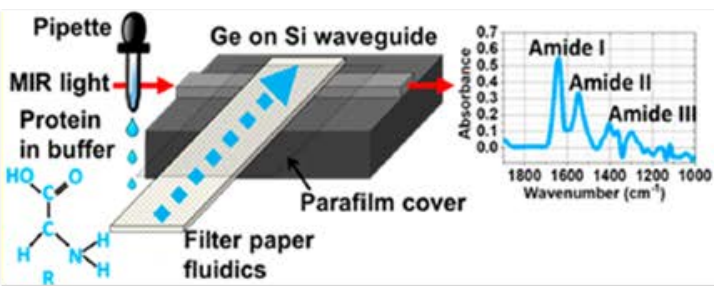

Figure 6 a) Scheme of the Ge waveguide spectroscopy sensor for liquids: a) a microfluidic chamber can be bonded onto the substrate, b) Image of the Ge waveguide bonded to the microfluidic channel: the microfluidic channel is $1 \mathrm{~mm}$ wide, $7 \mathrm{~mm}$ long, and $50 \mu \mathrm{m}$ deep, c) Germanium on Silicon waveguides showing BSA protein detection using filter paper as the microfluidic channel. Reprinted with permission from [56] Copyright (2018) Royal Society of Chemistry and [59] Copyright (2019) American Chemical Society respectively.

Diamond is a very robust and chemically inert material with a wide transparency window from the UV to the far-IR and a refractive index of 2.4. Although it is one of the most common materials used as an ATR crystal, its processing into thin film waveguides is challenging as single crystalline diamond films are currently available in mm scale pieces which can be used for thin film bonding technique but this is not ideal for large scale integrated circuits [52]. Polycrystalline diamond films have grain boundaries and a high density of defect centres due to lattice mismatch from the substrate, which are not suitable for low loss waveguides. One way to reduce the interfacial defects is to grow thicker films, as the film thickness increases the defect density reduces, and polish away the substrate to obtain low loss free standing films [51]. However, these films can be under high thermal residual stresses affecting their mechanical properties [53, 54]. Moreover, single and polycrystalline films of diamond are grown using chemical vapour deposition (CVD) where the substrate is kept above $700^{\circ} \mathrm{C}$ [55], which can be a problem to integrate with CMOS compatible processes.

\section{Germanium}

In, 2012, Ge strip waveguides on a Si substrate were integrated with a polymer based microfluidic cell to detect cocaine in tetrachloroethylene (PCE) solutions [56]. $2 \mu \mathrm{m}$ thick and $2.9 \mu \mathrm{m}$ wide Ge strip waveguides, single mode in the transverse magnetic (TM) polarization at $\lambda=5.8 \mu \mathrm{m}$, were fabricated using standard photolithography and reactive ion etching by fluorine chemistry. Light from a QCL emitting at $\lambda=5.8 \mu \mathrm{m}$ was coupled to a strip waveguide using ZnSe doublet lens, the output signal from the waveguide (after interacting with the liquid) was measured on an MCT detector using two parabolic mirrors. First, the real time measurement of water with different waveguide lengths was used to calibrate the response time of the sensor. Then real time measurements of different concentrations of Cocaine in PCE were studied with PCE as the background. The LOD of cocaine in PCE was reported to be $100 \mu \mathrm{g} / \mathrm{mL}$. The authors suggested that the LOD would be improved by reducing the system noise and using a longer waveguide. They proposed that the system noise due to alignment fluctuations can be reduced by using an integrated splitter with a reference arm, and coupling efficiency of the laser with the waveguide can be improved using the butt-coupling method. In 2013, the same group demonstrated a portable microsystem sensor based on waveguide spectroscopy to detect cocaine in human saliva using a droplet-based liquid-liquid extraction method to transfer cocaine from the IR absorbing (aqueous) saliva sample to IR transparent PCE [9]. A similar waveguide-QCL system as that in [56] was used. A polydimethylsiloxane (PDMS) microfluidic chamber was bonded onto a Ge strip waveguides as shown in Figure 6 (a) and (b) to demonstrate the first integration of a Ge waveguide with microfluidics. A concentration of $500 \mu \mathrm{g} / \mathrm{mL}$ of cocaine in an undiluted human saliva sample was successfully detected in real time using the optical waveguide system with on-chip extraction, which is sufficient for roadside testing of drugs. Later they improved the detection limit of cocaine in PCE to $10 \mu \mathrm{g} / \mathrm{mL}$ by increasing the contact area between the waveguide and the analyte by making the waveguide three times longer $[57,58]$. Since the absorbance of an analyte is directly proportional to the optical pathlength, increasing the pathlength directly increases the sensitivity and detection limit as long as the noise floor is not reached.

In 2018, spiral Ge waveguides on a silicon nitride (GON) platform and on a Si platform (GOS) were designed to detect isopropanol (IPA) in the wavelength region 3.65-3.90 $\mu \mathrm{m}$ [2]. The GON waveguides were fabricated by a wafer bonding and layer transfer technique and were $1.5 \mu \mathrm{m}$ thick, $1 \mu \mathrm{m}$ wide and $4.02 \mathrm{~mm}$ long, occupying an area of $260 \mu \mathrm{m} \times 260 \mu \mathrm{m}$. $1 \mu \mathrm{m}$ thick and $2 \mu \mathrm{m}$ wide GOS waveguides were fabricated on a crystalline Ge on Si wafer. A 3D printed PDMS microfluidic chamber was fabricated and sealed with the sensor chip. It was integrated with a syringe pump system for liquid handling. Several concentration mixtures of IPA with acetone were measured on both the waveguide platforms and the results were compared. The LOD of IPA in acetone was measured to be $5 \%$ for GON and $16 \%$ for GOS platforms.

In 2019, GOS waveguides were used to demonstrate for the first time all the three amide peaks in proteins in the wavelength region 5.3 to $10 \mu \mathrm{m}\left(1900-1000 \mathrm{~cm}^{-1}\right)$ [59]. A paper fluidic technique was used to introduce the liquid analyte onto the waveguide surface, as in [45], as shown in Figure 6 (c). A QCL was used as a light source and ZnSe lenses for both input and output free-space coupling. A thermo-electrically (TE) cooled MCT detector was used to measure the output signal. A concentration series of bovine serum albumin (BSA) mixed in phosphate buffer saline (PBS) from $0.1 \mathrm{mg} / \mathrm{mL}$ to $100 \mathrm{mg} / \mathrm{mL}$ was measured on the waveguide. Subsequently, using the same GOS waveguide-filter paper-QCL system as reported in [59], measurement of BSA protein's monomer, oligomer and 
(a)

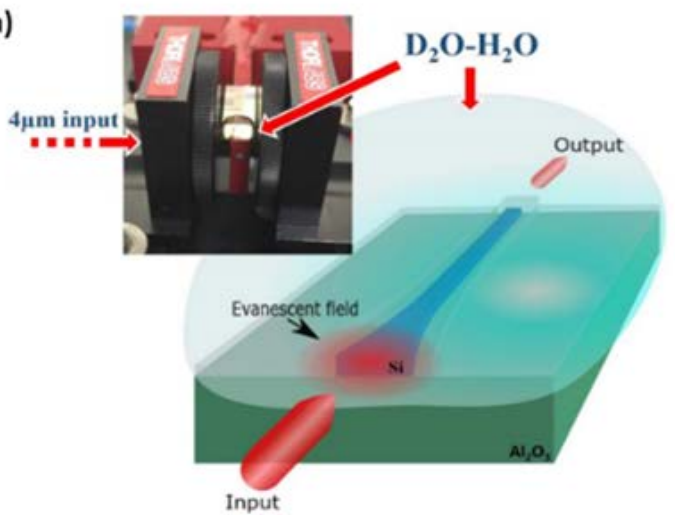

(b)

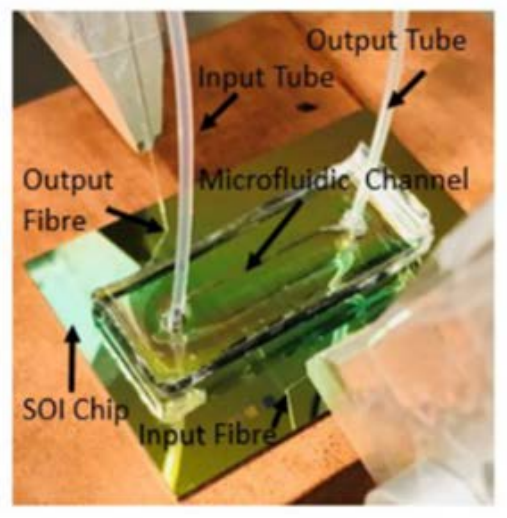

Figure 7 a) Layout of a SOS sensor cladded with the sample liquid; the evanescent field of the guided mode interacts with the liquid, which is utilized for sensing (Inset: image of the SOS chip with a $\mathrm{D}_{2} \mathrm{O}-\mathrm{H}_{2} \mathrm{O}$ mixture), b) SOI chip with microfluidics and pump tubes. Reprinted with permission from [61] (C) The Optical Society (2016) and [3] SPIE (2019) respectively.

fibrils prepared in DI water were demonstrated [60]. A clear shift in the structures of BSA protein and its aggregates was observed in amide I and II peak positions and in their peak shapes corresponding to changes in protein conformations. These results confirm the potential for distinguishing monomers from toxic oligomers and fibrils using MIR waveguide spectroscopy.

Ge is transparent in the IR region up to $16 \mu \mathrm{m}$ and has a refractive index of $\sim 4$, though it is brittle and requires careful handling. The high refractive index causes high reflection losses from input and output facets. Ge waveguides typically have a short penetration depth because of their high refractive index so $\mathrm{Ge}$ is suitable for application to highly sensitive spectroscopy of ultrathin films.

\section{Silicon (Si)}

CMOS compatible silicon on sapphire (SOS) strip waveguides were used for sensing of heavy water $\left(\mathrm{D}_{2} \mathrm{O}\right)$ in water $\left(\mathrm{H}_{2} \mathrm{O}\right)$ as shown in Figure 7 (a) [61]. Light from a QCL source emitting at $\lambda=3.94 \mu \mathrm{m}$ was coupled into a $3 \mu \mathrm{m}$ wide and $500 \mu \mathrm{m}$ thick multimode SOS waveguide using a chalcogenide glass lens. A $35 \mu \mathrm{L}$ drop of $\mathrm{D}_{2} \mathrm{O}-\mathrm{H}_{2} \mathrm{O}$ mixture was introduced onto the waveguide surface using a syringe. The output signal was recorded on a cooled InSb detector and the cross-section of the waveguide output was imaged on an IR camera. The data and images were recorded for concentrations ranging from $0.25 \%$ to $12 \%$ before and after applying the liquid sample. The LOD of $\mathrm{D}_{2} \mathrm{O}$ was found to be $0.25 \%$ (2500ppmv). Si pedestal waveguides were designed and fabricated for on chip sensing of organic solvents in the MIR region of $\lambda=2.5$ to $3.7 \mu$ m [62]. Concentrations of toluene in carbon tetrachloride $\left(\mathrm{CCL}_{4}\right)$ ranging from 0 to 1 weight ratio were measured at $\lambda=3.3 \mu \mathrm{m}$ using the strong absorption of toluene at this wavelength from the aromatic C-H stretching vibration. Screening of organic solvents such as methanol, toluene, hexane, water, $\mathrm{CCL}_{4}$ and acetone was carried out by measuring each solvent at $\lambda=$ $3.55 \mu \mathrm{m}$ and their relative absorbance was compared to distinguish each chemical at their absorption wavelength.
In 2019, silicon on insulator (SOI) waveguides combined with PDMS microfluidics, as shown in Figure 7 (b), were used to study the LOD of IPA in water at wavelengths between 3.725$3.88 \mu \mathrm{m}$ [3]. Single mode SOI waveguides, 500 $\mathrm{nm}$ thick and $1.3 \mu \mathrm{m}$ wide, with lengths from $10 \mu \mathrm{m}$ to $2 \mathrm{~cm}$ were fabricated. A QCL was used as a light source and fluoride fibers were used to couple the light via grating couplers. A liquid nitrogen cooled InSb detector was used to record the spectrum. A syringe pump system was used with PDMS microfluidics. LOD of $3 \%$ IPA in water was achieved at $\lambda=3.77 \mu \mathrm{m}$.

$\mathrm{Si}$ is a hard material and chemically inert to most materials except strong acids. It is transparent in the MIR up to $8 \mu \mathrm{m}$ and has a high refractive index of 3.4-3.5. Typically SOI is used for sensing in the MIR, however, the silica substrate's high absorption losses beyond $\lambda=3.7 \mu \mathrm{m}$ limits its capability to utilize the full Si transparency window. To overcome this problem, air-clad suspended $\mathrm{Si}$ waveguides were proposed $[63,64] . \mathrm{Si}_{3} \mathrm{~N}_{4}$ is transparent from visible wavelengths up to $8.5 \mu \mathrm{m}[65]$ and has a refractive index of $\sim 2$, depending on the stoichiometry between Si and N. It is chemically resistant and biocompatible [66]. The most common substrate used for $\mathrm{Si}_{3} \mathrm{~N}_{4}$ deposition is silica which again limits its use in the MIR region beyond $3.7 \mu \mathrm{m}$ of wavelength. However, suspended $\mathrm{Si}_{3} \mathrm{~N}_{4}$ ring resonators have been demonstrated for optical sensing at telecommunication wavelengths of 1.3 and $1.55 \mu \mathrm{m}$ [67].

Group IV materials such as $\mathrm{Ge}, \mathrm{Si}$ and $\mathrm{Si}_{3} \mathrm{~N}_{4}$ benefit from CMOS compatible, standard and mature fabrication processes. Among the material systems discussed, group IV materials appear the most promising given their high refractive index, standard fabrication and processing, large MIR transparency, and chemical and biochemical compatibility. 
Table 1. Liquid analytes measured on waveguide systems in the MIR

\begin{tabular}{|c|c|c|c|c|c|c|}
\hline $\begin{array}{l}\text { Waveguide } \\
\text { system }\end{array}$ & $\begin{array}{l}\text { Frequency/ } \\
\text { Wavelength region } \\
\text { of transparency } \\
\left(\mathrm{cm}^{-1} / \mu \mathrm{m}\right)\end{array}$ & $\begin{array}{l}\text { Frequency/ } \\
\text { Wavelength region } \\
\text { of measurement } \\
\left(\mathrm{cm}^{-1} / \mu \mathrm{m}\right)\end{array}$ & Analyte measured & $\begin{array}{l}\text { Analyte } \\
\text { volume }\end{array}$ & $\begin{array}{l}\text { LOD } \\
\text { measured }\end{array}$ & Ref \\
\hline $\begin{array}{l}\text { Silver hal- } \\
\text { ide }\end{array}$ & 3333.3-500 / 3-20 & 974 / 10.3 & $\begin{array}{l}\text { Acetic anhydride in acetoni- } \\
\text { trile }\end{array}$ & $1 \mu \mathrm{L}$ & $\begin{array}{l}0.01 \mu \mathrm{L} \\
(10.8 \mathrm{mg} / \mathrm{mL})\end{array}$ & [29] \\
\hline \multirow{4}{*}{ GaAs } & \multirow{4}{*}{ 10000-666.7 / 1-15 } & $974 / 10.3$ & Acetic anhydride & $2 n L$ & $\begin{array}{l}0.05 \mathrm{pL} \\
\left(5.29 \times 10^{-4}\right. \\
\mathrm{mg} / \mathrm{mL})\end{array}$ & [32] \\
\hline & & $1100 / 9.09$ & $\begin{array}{l}\text { Ammonium perchlorate in } \\
\text { water }\end{array}$ & $50 \mu \mathrm{L}$ & $\begin{array}{l}56.9 \mathrm{mM} \\
(6.68 \mathrm{mg} / \mathrm{mL})\end{array}$ & [34] \\
\hline & & $1700 / 5.88$ & Benzaldehyde in DGME & & $\begin{array}{l}35.1 \mathrm{mM} \\
(3.72 \mathrm{mg} / \mathrm{mL})\end{array}$ & [34] \\
\hline & & $910 / 10.98$ & $\begin{array}{l}\text { Perchloroethylene in DI } \\
\text { water }\end{array}$ & & $\begin{array}{l}\text { 5ppm } \\
(0.005 \mathrm{mg} / \mathrm{mL})\end{array}$ & [35] \\
\hline MCT & $4000-500 / 2.5-20$ & $1570-1735$ / 5.78-6.35 & Acetone in IPA & $0.2 \mu \mathrm{L}$ & $\begin{array}{l}1 \mathrm{~nL} \\
(3.96 \mathrm{mg} / \mathrm{mL})\end{array}$ & [39] \\
\hline GeAsSe & $\begin{array}{l}4000-1515.15 / 2.5- \\
6.6\end{array}$ & 3333- 1333 / 3-7.5 & Prussian blue in DMSO & & $\begin{array}{l}0.1 \mathrm{wt} \% \\
(0.01 \mathrm{mg} / \mathrm{ml})\end{array}$ & [40] \\
\hline ZnSe & $\begin{array}{l}16666.6-625 / 0.6- \\
16\end{array}$ & $3846-2702$ / 2.6-3.7 & IPA in water & & $\begin{array}{l}20 \% * \\
(200 \mathrm{mg} / \mathrm{mL})\end{array}$ & [45] \\
\hline \multirow[t]{3}{*}{ Diamond } & \multirow[t]{3}{*}{$\begin{array}{l}33333.3-4000 / 0.3- \\
2.5, \\
1428.57-100 / 7-100\end{array}$} & $1710 / 5.8$ & Acetone in $\mathrm{D}_{2} \mathrm{O}$ & $5 \mu \mathrm{L}$ & $\begin{array}{l}200 \mathrm{pL} \\
(52.7 \mathrm{mg} / \mathrm{mL})\end{array}$ & [49] \\
\hline & & $1700-1600 / 5.88-6.25$ & Protein & $5 \mu \mathrm{L}$ & $10 \mathrm{mg} / \mathrm{mL}^{*}$ & [50] \\
\hline & & $1650 / 6.06$ & DMF in water & $50 \mu \mathrm{L}$ & $\begin{array}{l}5 \mathrm{vol} \% \\
(0.5 \mathrm{mg} / \mathrm{ml})\end{array}$ & [51] \\
\hline \multirow{4}{*}{ Ge } & \multirow{4}{*}{$5000-625$ / 2-16 } & $1710 / 5.8$ & Cocaine in saliva & & $\begin{array}{l}500 \mu \mathrm{g} / \mathrm{mL} \\
(0.5 \mathrm{mg} / \mathrm{mL})\end{array}$ & [9] \\
\hline & & $1710 / 5.8$ & Cocaine in PCE & & $\begin{array}{l}10 \mu \mathrm{g} / \mathrm{mL} \\
(0.01 \mathrm{mg} / \mathrm{mL})\end{array}$ & $\begin{array}{l}{[57,} \\
58]\end{array}$ \\
\hline & & $1900-1000 / 2.26-10$ & Protein in PBS & & $0.1 \mathrm{mg} / \mathrm{mL}^{*}$ & [59] \\
\hline & & 2739-2564/ 3.65-3.90 & IPA in acetone & & $\begin{array}{l}5 \% \\
(50 \mathrm{mg} / \mathrm{mL})\end{array}$ & {$[2]$} \\
\hline \multirow[t]{2}{*}{$\mathrm{Si}$} & \multirow[t]{2}{*}{$8333.3-1250$ / 1.2-8 } & 2538 / 3.94 & $\mathrm{D}_{2} \mathrm{O}$ in water & $35 \mu \mathrm{L}$ & $\begin{array}{l}0.25 \% \\
(2.5 \mathrm{mg} / \mathrm{mL})\end{array}$ & [61] \\
\hline & & $\begin{array}{l}2684-2577 \text { / 3.725- } \\
3.88\end{array}$ & IPA in water & & $\begin{array}{l}3 \% \\
(30 \mathrm{mg} / \mathrm{mL})\end{array}$ & [3] \\
\hline
\end{tabular}

* In these publications LOD was not stated; the values show the minimum concentration measured

\section{$\begin{array}{llll}\text { OUTLOOK } & \text { AND } & \text { FUTURE } & \text { TRENDS }\end{array}$}

While significant advances in MIR waveguide materials and devices have been made in recent years, the development of other components and tools required to enable MIR spectroscopy in applications related to environmental sensing and early diagnostic of diseases has also advanced, improving the prospects for adoption of MIR waveguide spectroscopy. The development of high power tunable light sources, highly sensitive detectors, compact and light-weight instruments, high resolution data acquisition methods, and of spectral data processing software are some examples. More specifically, a sig- 
nificant improvement in S/N has been achieved using waveguides combined with QCLs as compared to a commercial FTIR [49, 68]. MIR spectroscopy promises rapid screening for specific diseases such as kidney, neurological, respiratory, urinary tract, gastro-intestinal, metabolic, genetic, gynaecological and dermatological disorders [69-73], but at present it remains a research technique. Body fluids are complex and different for each individual so that comprehensive databases correlating spectral measurements with pathology or chemical composition will be required for reliable diagnosis. While high-quality measurements and reference data are of fundamental importance, robust chemometric techniques for data treatment, base-line removal, spectral deconvolution, normalization and noise-reduction will need to be adopted [74, 75].

Before MIR spectroscopy becomes a mainstream clinical tool, standardization and extensive clinical trials needs to be carried out. Absorption spectroscopy is a promising technique that can be combined with advanced thin film waveguide technology, powerful QCL sources, detectors and chemometric algorithms or machine learning approaches that can provide accurate analysis of the data to probe the body fluids directly without extensive processing of the samples for a quick and reliable outcome. On-chip spectroscopy allows integration of multiple functionalities such as microfluidics and electronics on the same photonic chip, yielding enhanced stability, miniaturization and compactness. Multiplexing and the ability to simultaneously measure multiple samples is possible using waveguide technology as compared to ATR, due to the ease of integrating multiple devices on a chip using the fabrications approaches developed for microelectronics. It is expected that careful device design combined with surface concentration strategies will enable sensitive measurement of biochemical species in aqueous media.

\section{AUTHOR INFORMATION}

Corresponding Author

Vinita Mittal

*Email: V.Mittal@soton.ac.uk

Notes

The authors declare no competing financial interest.

\section{ACKNOWLEDGMENT}

The authors would like to acknowledge the EPSRC ElectronicPhotonic Convergence Platform grant (EP/N013247/1).

\section{REFERENCES}

1. Parachalil, D.R.; Bruno, C.; Bonnier, F.; Blasco, H.; Chourpa, I.; Baker, M.J.; McIntyre, J.; Byrne, H.J. Analysis of bodily fluids using vibrational spectroscopy: a direct comparison of Raman scattering and infrared absorption techniques for the case of glucose in blood serum. Analyst 2019, 144(10), pp.3334-3346.

2. Li, W.; Anantha, P.; Lee, K.H.; Qiu, H.D.; Guo, X.; Goh, S.C.K.; Zhang, L.; Wang, H.; Soref, R.A.; Tan, C.S. Spiral wave-guides on germanium-on-silicon nitride platform for mid-IR sensing applications. IEEE Photonics J. 2018, 10(3), pp.1-7.

3. Qi, Y.; Rowe, D. J.; Mittal, V.; Banakar, M.; Wu, Y.; Nedeljković, M.; Wilkinson, J. S.; Mashanovich, G. Z. Integration of mid-infrared SOI photonics with microfluidics. Reed, Graham T. and Knights, Andrew P. (eds.) In Silicon Photonics XIV. 2019, vol. 10923, SPIE. 9 (doi:10.1117/12.2507815).
4. Brandstetter, M.; Volgger, L.; Genner, A.; Jungbauer, C.; Lendl, B. Direct determination of glucose, lactate and triglycerides in blood serum by a tunable quantum cascade laser-based mid-IR sensor. Appl. Phys. B 2013, 110(2), pp.233-239.

5. Haas, J.; Müller, A.; Sykora, L.; Mizaikoff, B. Analytical performance of $\mu$-groove silicon attenuated total reflection waveguides. Analyst 2019, 144(10), pp.3398-3404.

6. Haas, J.; Catalán, E.V.; Piron, P.; Karlsson, M.; Mizaikoff, B. Infrared spectroscopy based on broadly tunable quantum cascade lasers and polycrystalline diamond waveguides. Analyst 2018, 143(21), pp.5112-5119.

7. Moorthy, B. S.; Iyer, L. K.; Topp E. M. Characterizing protein structure, dynamics and conformation in lyophilized solids. Curr. Pharm. Des. 2015, 21(40), pp.5845-5853.

8. Odoli, C. O.; Oduor-Odote P.; Arason, S. The influence of lipid content and pre-treatment methods on protein conformation in fish (capelin, Mallotus villosus) during smoking and drying. J. Food Sci. Nutr. 2019, 7(4), pp.1446-1454.

9. Wägli, P.; Chang, Y.C.; Homsy, A.; Hvozdara, L.; Herzig, H.P.; de Rooij, N.F. Microfluidic droplet-based liquid-liquid extraction and on-chip IR spectroscopy detection of cocaine in human saliva. Anal. Chem. 2013, 85(15), pp.7558-7565.

10. Tao, L.; Lin, Z.; Chen, J.; Wu, Y.; Liu, X. Mid-infrared and nearinfrared spectroscopy for rapid detection of Gardeniae Fructose by a liquid-liquid extraction process. J. Pharmaceut. Biomed. 2017, 145, pp.1-9.

11. Rigler, P.; Ulrich, W.P.; Hoffmann, P.; Mayer, M.; Vogel, H. Reversible immobilization of peptides: surface modification and in situ detection by attenuated total reflection FTIR spectroscopy. ChemPhysChem. 2003, 4(3), pp.268-275.

12. Janotta, M.; Mizaikoff, B. March. Recent developments in liquid phase mid-infrared sensor technology. In Optical Fibers and Sensors for Medical Applications II 2002, vol. 4616, pp. 1-8 International Society for Optics and Photonics.

13. Biegert, J.; Bates, P.K.; Chalus, O. New mid-infrared light sources. IEEE J. Sel. Top. Quant. 2011, 18(1), pp.531-540.

14. Faist, J. Quantum cascade lasers. 2013, OUP Oxford.

15. Haas, J.; Mizaikoff, B. Advances in mid-infrared spectroscopy for chemical analysis. Annu. Rev. Anal. Chem. 2016, 9, pp.45-68.

16. Jung, D.; Bank, S.; Lee, M.L.;Wasserman, D. Next-generation midinfrared sources. J. Opt. 2017, 19(12), pp.123001.

17. Keen, J.A.; Lane, D.; Kesaria, M.; Marshall, A.R.J.; Krier, A. InAs/InAsSb type-II strained-layer superlattices for mid-infrared LEDs. J. Phys. D Appl. Phys. 2018, 51(7), pp.075103.

18. Karim, A.; Andersson, J.Y. Infrared detectors: Advances, challenges and new technologies. In IOP Conference Series: Mater. Sci. and Eng. 2013, 51, (1) pp. 012001.

19. Piotrowski, J. Infrared Detectors-New Trends. Acta Physica Polonica-Series A Gen. Phy. 1995, 87(2), pp.303-316.

20. Vigneron, P.B.; Pirotta, S.; Carusotto, I.; Tran, N.L.; Biasiol, G.; Manceau, J.M.; Bousseksou, A.; Colombelli, R. Quantum well infrared photo-detectors operating in the strong light-matter coupling regime. Appl. Phys. Lett. 2019, 114(13), p.131104.

21. Delga, A. Quantum cascade detectors: A review. In Mid-infrared Optoelectronics 2020, pp. 337-377. Woodhead Publishing.

22. Ren, A.; Yuan, L.; Xu, H.; Wu, J.; Wang, Z. Recent progress of III$\mathrm{V}$ quantum dot infrared photodetectors on silicon. J. Mater.Chem. C 2019, 7(46), pp.14441-14453.

23. Hu, J.; Shi, Y.; Zhang, Z.; Zhi, R.; Yang, S.; Zou, B. Recent progress of infrared photodetectors based on lead chalcogenide colloidal quantum dots. Chin. Phys. B. 2019, 28(2), p.020701.

24. Müller, R.; Niemasz, J.; Daumer, V.; Janaszek, A.; Jureńczyk, J.; Rehm, R. Advances on photoconductive InAs/GaSb type-II superlattice long-wavelength infrared detectors for high operating temperature. In Optical Components and Materials XVI 2019 February, vol. 10914, pp. 1091416. International Society for Optics and Photonics.

25. https://www.thorlabs.com/newgrouppage9.cfm?objectgroup id=706 2\#ad-image-0

26. https://irflex.com/products/irf-se-series/

27. https://www.optoknowledge.com/mid-ir-fiber-opticscoating.html\#: :text=Mid\%2DIR\%20Hollow\%20Fibers,\%3A\%20\%CE \%BB\%20\%3D\%202\%20\%E2\%80\%93\%2016\%20\%C2\%B5m 
28. https://www.edmundoptics.co.uk/c/ir-lenses/655/

29. Charlton, C.; Katzir, A.; Mizaikoff, B. Infrared evanescent field sensing with quantum cascade lasers and planar silver halide waveguides. Anal. Chem. 2005, 77(14), pp.4398-4403.

30. Schädle, T.; Eifert, A.; Kranz, C.; Raichlin, Y.; Katzir, A.; Mizaikoff, B. Mid-infrared planar silver halide waveguides with integrated grating couplers. Appl. Spectrosc. 2013, 67(9), pp.1057-1063.

31. Charlton, C.; Giovannini, M.; Faist, J.; Mizaikoff, B. Fabrication and characterization of molecular beam epitaxy grown thin-film GaAs waveguides for mid-infrared evanescent field chemical sensing. Anal. Chem. 2006, 78(12), pp.4224-4227.

32. Wang, X.; Kim, S.S.; Roßbach, R.; Jetter, M.; Michler, P.; Mizaikoff, B. Ultra-sensitive mid-infrared evanescent field sensors combining thin-film strip waveguides with quantum cascade lasers. Analyst 2012, 137(10), pp.2322-2327.

33. Sieger, M.; Balluff, F.; Wang, X.; Kim, S.S.; Leidner, L.; Gauglitz, G.; Mizaikoff, B. On-chip integrated mid-infrared GaAs/AlGaAs Mach-Zehnder interferometer. Anal. Chem. 2012, 85(6), pp.30503052.

34. Sieger, M.; Haas, J.; Jetter, M.; Michler, P.; Godejohann, M.; Mizaikoff, B. Mid-infrared spectroscopy platform based on GaAs/AlGaAs thin-film waveguides and quantum cascade lasers. Anal. Chem. 2016, 88(5), pp.2558-2562.

35. Haas, J.; Stach, R.; Sieger, M.; Gashi, Z.; Godejohann, M.; Mizaikoff, B. Sensing chlorinated hydrocarbons via miniaturized GaAs/AlGaAs thin-film waveguide flow cells coupled to quantum cascade lasers. Anal. Methods 2016, 8(36), pp.6602-6606.

36. Sieger, M.; Kos, G.; Sulyok, M.; Godejohann, M.; Krska, R.; Mizaikoff, B. Portable infrared laser spectroscopy for on-site mycotoxin analysis. Sci. Rep. 2017, 7, p.44028.

37. Cho, Y.; Ivanisevic, A. In vitro assessment of the biocompatibility of chemically modified GaAs surfaces. J. NanoBiotechnology. 2006, 2(1-2), pp.51-59.

38. Liebl, B.; Mueckter, H.; Nguyen, P.T.; Doklea, E.; Islambouli, S.; Fichtl, B.; Forth, W. Differential effects of various trivalent and pentavalent organic and inorganic arsenic species on glucose metabolism in isolated kidney cells. Appl. Organometallic Chem. 1995, 9(7), pp.531-540.

39. Wang, X.; Antoszewski, J.; Putrino, G.; Lei, W.; Faraone, L.; Mizaikoff, B. Mercury-cadmium-telluride waveguides-a novel strategy for on-chip mid-infrared sensors. Anal. Chem. 2013, 85(22), pp.10648-10652.

40. Ma, P.; Choi, D.Y.; Yu, Y.; Gai, X.; Yang, Z.; Debbarma, S.; Madden, S.; Luther-Davies, B. Low-loss chalcogenide waveguides for chemical sensing in the mid-infrared. Opt. Exp. 2013, 21(24), pp.29927-29937.

41. Baudet, E.; Gutierrez-Arroyo, A.; Baillieul, M.; Charrier, J.; Němec, P.; Bodiou, L.; Lemaitre, J.; Rinnert, E.; Michel, K.; Bureau, B.; Adam, J.L. Development of an evanescent optical integrated sensor in the mid-infrared for detection of pollution in groundwater or seawater. Adv. Device Mater. 2017, 3(2), pp.23-29.

42. Gutierrez-Arroyo, A.; Baudet, E.; Bodiou, L.; Nazabal, V.; Rinnert, E.; Michel, K.; Bureau, B.; Colas, F.; Charrier, J. Theoretical study of an evanescent optical integrated sensor for multipurpose detection of gases and liquids in the mid-infrared. Sensors Actuat. B Chem. 2017, 242, pp.842-848.

43. Nam, C.; Zimudzi, T.J.; Wiencek, R.A.; Chung, T.M.; Hickner, M.A. Improved ATR-FTIR detection of hydrocarbons in water with semi-crystalline polyolefin coatings on ATR elements. Analyst 2018, 143(22), pp.5589-5596.

44. Singh, V.; Lin, P.T.; Patel, N.; Lin, H.; Li, L.; Zou, Y.; Deng, F.; Ni, C.; Hu, J.; Giammarco, J.; Soliani, A.P. Mid-infrared materials and devices on a Si platform for optical sensing. Sci. and Technol. Adv. Mat. 2014, 15(1), p.014603.

45. Mittal, V.; Nedeljković, M.; Rowe, D.; Murugan, G.S.; Wilkinson, J. $\mathrm{S}$. Chalcogenide glass waveguides with paper-based fluidics for midinfrared absorption spectroscopy, Opt. Lett. 2018, 43, pp.2913-2916.

46. https://www.bruker.com/fileadmin/user_upload/8-PDFDocs/OpticalSpectrospcopy/FT-IR/ALPHA/AN/AN79_ATRBasics_EN.pdf

47. https://www.news-medical.net/health/pH-in-the-Human-Body.aspx

48. https://www.livestrong.com/article/442195-the-overall-ph-of-bodyfluid/
49. Wang, X.; Karlsson, M.; Forsberg, P.; Sieger, M.; Nikolajeff, F.; Österlund, L.; Mizaikoff, B. Diamonds are a spectroscopist's best friend: thin-film diamond mid-infrared waveguides for advanced chemical sensors/biosensors. Anal. Chem. 2014, 86(16), pp.81368141.

50. López-Lorente, Á.I.; Wang, P.; Sieger, M.; Vargas Catalan, E.; Karlsson, M.; Nikolajeff, F.; Österlund, L.; Mizaikoff, B. Midinfrared thin-film diamond waveguides combined with tunable quantum cascade lasers for analyzing the secondary structure of proteins. Phys. Status Solidi A 2016, 213(8), pp.2117-2123.

51. Haas, J.; Catalán, E.V.; Piron, P.; Nikolajeff, F.; Österlund, L.; Karlsson, M.; Mizaikoff, B. Polycrystalline diamond thin-film waveguides for mid-infrared evanescent field sensors. ACS omega, 2018, 3(6), pp.6190-6198.

52. Hill, P.; Gu, E.; Dawson, M.D.; Strain, M.J. Thin film diamond membranes bonded on-demand with SOI ring resonators. Diam. Relat. Mater. 2018, 88, pp.215-221.

53. Feng, C.H.E.N.; Gen, X.U.; Xu, G.P. Thermal residual stress of polycrystalline diamond compacts. T. Nonferr. Metal. Soc.of China 2010, 20(2), pp.227-232.

54. Harker, A.B.; Howitt, D.G.; Chen, S., Flintoff; J.F.; James, M.R. Residual stress measurements on polycrystalline diamond. Window and Dome Technologies and Materials IV September 1994, 2286, pp. 254-261 International Society for Optics and Photonics.

55. May, P.W. Diamond thin films: a 21st-century material. Philos. Trans. Royal Soc. A: Mathematical, Physical and Engineering Sciences, 2000, 358(1766), pp.473-495.

56. Chang, Y.C.; Wägli, P.; Paeder, V.; Homsy, A.; Hvozdara, L.; van der Wal, P.; Di Francesco, J.; de Rooij, N.F.; Herzig, H.P. Cocaine detection by a mid-infrared waveguide integrated with a microfluidic chip. Lab Chip 2012, 12(17), pp.3020-3023.

57. Jouy, P.; Mangold, M.; Tuzson, B.; Emmenegger, L.; Chang, Y.C.; Hvozdara, L.; Herzig, H.P.; Wägli, P.; Homsy, A.; de Rooij, N.F.; Wirth-mueller, A.; Hofstetter D.; Losser H.; Faist J.. Mid-infrared spectroscopy for gases and liquids based on quantum cascade technolo-gies. Analyst 2014, 139(9), pp.2039-2046.

58. Wagli, P. PhD Thesis, Ecole Polytechnique F'ed'erale de Lausanne, December 2012.

59. Mittal, V. ; Nedeljkovic, M.; Carpenter, L. G.; Khokhar, A. Z.; Chong, H. M. H.; Mashanovich, G. Z.; Bartlett, P. N.; Wilkinson, J. S. Waveguide absorption spectroscopy of bovine serum albumin in the mid-infrared fingerprint region. ACS Sens. 2019, 4 (7), pp.17491753

60. Mittal, V.; Devitt, G.; Bartlett, P. N.; Chong, H. M. H.; Mashanovich, G.; Mahajan S.; Wilkinson, J. S. Mid-infrared absorption spectroscopy of protein aggregates using germanium on silicon waveguides', ECIO 2019, Ghent, Belgium, 24-26 April 2019.

61. Singh, N.; Casas-Bedoya, A.; Hudson, D.D.; Read, A.; Mägi, E.; Eggleton, B.J. Mid-IR absorption sensing of heavy water using a silicon-on-sapphire waveguide. Opt. Lett. 2016, 41(24), pp.5776-5779.

62. Lin, P.T.; Singh, V.; Hu, J.; Richardson, K.; Musgraves, J.D.; Luzinov, I.; Hensley, J.; Kimerling, L.C.; Agarwal, A. Chip-scale midinfrared chemical sensors using air-clad pedestal silicon waveguides. Lab Chip. 2013, 13(11), pp.2161-2166.

63. Penadés, J.S.; Alonso-Ramos, C.; Khokhar, A.Z.; Nedeljkovic, M.; Boodhoo, L. A.; Ortega-Moñux, A.; Molina-Fernández, I; Cheben, P.; Mashanovich, G. Z. Suspended SOI waveguide with subwavelength grating cladding for mid-infrared, Opt. Lett. 2014, 39, 5661-5664.

64. Penadés, J.S.; Sánchez-Postigo, A.; Nedeljkovic, M.; Ortega-Moñux, A.; Wangüemert-Pérez, J.G.; Xu, Y.; Halir, R.; Qu, Z.; Khokhar, A.Z.; Osman, A.; Cao, W. Suspended silicon waveguides for longwave infrared wavelengths. Opt. Lett. 2018, 43(4), pp.795-798.

65. Tai Lin, P.; Singh, V.; Kimerling, L.; Murthy Agarwal, A. Planar silicon nitride mid-infrared devices. Appl. Phys. Lett. 2013, 102(25), pp.251121.

66. Neumann A.; Reske T.; Held M, Jahnke, K.; Ragoss, C.; Maier, H.R Comparative investigation of the biocompatibility of various silicon nitride ceramic qualities in vitro. J. of Mater. Sci.: Materials in Medicine. 2004, 15(10):1135-40.

67. Feng, J.; Akimoto, R. T-shape suspended silicon nitride ring resonator for optical sensing applications. IEEE Photon. Tech. Lett. 2015, 27(15), pp.1601-1604 
68. Schädle, T.; Mizaikoff, B. Mid-infrared waveguides: a perspective. Appl. Spectrosc. 2016, 70(10), pp.1625-1638.

69. Paraskevaidi, M.; Morais, C.L.; Lima, K.M.; Snowden, J.S.; Saxon, J.A.; Richardson, A.M.; Jones, M.; Mann, D.M.; Allsop, D.; MartinHirsch, P.L.; Martin, F.L. Differential diagnosis of Alzheimer's disease using spectrochemical analysis of blood. PNAS 2017, 114(38), pp.E7929-E7938.

70. Balan, V.; Mihai, C.T.; Cojocaru, F.D.; Uritu, C.M.; Dodi, G.; Botezat, D.; Gardikiotis, I. Vibrational spectroscopy fingerprinting in medicine: from molecular to clinical practice. Materials. 2019, 12(18), p.2884.

71. Ghimire, H.; Venkataramani, M.; Bian, Z.; Liu, Y.; Perera, A.U. ATR-FTIR spectral discrimination between normal and tumorous mouse models of lymphoma and melanoma from serum samples. Sci. Rep. 2017, 7(1), pp.1-9.

72. Li, R.; Rui, G.; Chen, W.; Li, S.; Schulz, P.E.; Zhang, Y. Early detection of Alzheimer's disease using non-invasive near-infrared spectroscopy. Front. Aging Neurosci. 2018, 10, p.366.

73. Rodrigues, R.P.; Aguiar, E.M.; Cardoso-Sousa, L.; Caixeta, D.C.; Guedes, C.C.; Siqueira, W.L.; Maia, Y.C.P.; Cardoso, S.V.; SabinoSilva, R. Differential molecular signature of human saliva using ATR-FTIR spectroscopy for chronic kidney disease diagnosis. Braz. Dent. J. 2019, 30(5), pp.437-445.

74. Mwanga, E.P.; Minja, E.G.; Mrimi, E.; Jiménez, M.G.; Swai, J.K.; Abbasi, S.; Ngowo, H.S.; Siria, D.J.; Mapua, S.; Stica, C.; Maia, M.F. Detection of malaria parasites in dried human blood spots using mid-infrared spectroscopy and logistic regression analysis. Malaria J. 2019, 18(1), pp.1-13.

75. De Bruyne, S.; Speeckaert, M.M.; Delanghe, J.R. Applications of mid-infrared spectroscopy in the clinical laboratory setting. Crit. Rev. Cl. Lab Sci. 2018, 55(1), pp.1-20.

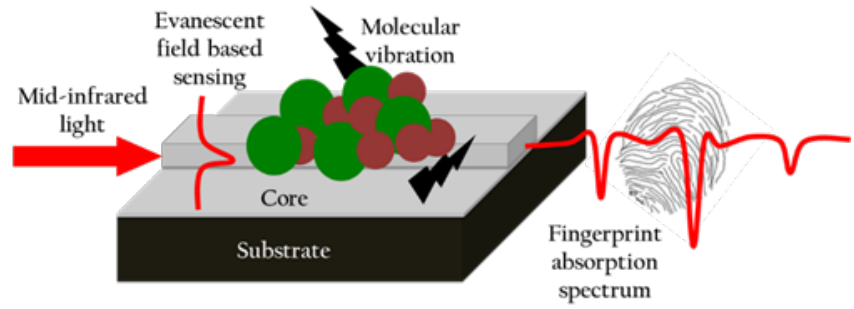

